\title{
Predicting Dementia in the Elderly: a Physician-friendly Formula
}

In this issue, Hogan $\&$ Ebly $^{1}$ publish the results of a study designed to see if clinically pragmatic variables obtainable at first medical contact could be useful in predicting dementia in non-demented elderly Canadians who were being followed five years later through the Canadian Study of Health and Aging $(\mathrm{CSHA}){ }^{2}$ A real strength is that this is a population-based national survey of a representative sample of Canadians over age 65 , including both community and institutional dwellers. Part 1 of the CSHA study in 1990-1991 established solid prevalence figures for dementia in Canada. ${ }^{2}$ The second wave of this study, in which 1782 non-demented individuals were comprehensively reassessed, is particularly important because it is free of the bias inherent in referral clinics. Although $40 \%$ attrition of the sample occurred due to death, the numbers are still sizable, with 240 individuals of the 892 survivors being diagnosed as having dementia at follow-up. For this particular prognostication, the authors deliberately sought to use information easily obtainable by a physician in the office setting. A mental status instrument, the modified Mini Mental State Examination (mMMSE) ${ }^{3}$ was used because it has better sensitivity and specificity for detecting dementia than the Folstein MMSE, ${ }^{4}$ the items for which are nevertheless contained within the mMMSE. Age, sex, education, informant history of memory problems, family history of dementia, and competence in activities of daily living were also obtained as potential predictor variables. The results are clinically useful and could help physicians determine who should be followed more closely or further investigated for incipient dementia. This is particularly relevant with the advent of new potential therapies.

Logistic regression was employed to determine sensitivity and specificity and to derive a simple algorithm for predicting risk. The simplest model, including the MMSE or mMMSE score (either one worked), age and an informant's report of memory problem, gave a sensitivity of around $80 \%$ and specificity of $56 \%$ for predicting dementia. The authors break down the predictive model for non-cognitively impaired as well as cognitive impairment without dementia (CIND) subjects, and looked at prediction of dementia from any cause as well as from Alzheimer's Disease (AD). The resulting equation provides a handy formula for physicians to calculate the probability of dementia developing and can guide further investigation and planning accordingly. A disadvantage of using the mMMSE is that it is not widely used in the physician community and it does take more time to administer. Happily, substituting the MMSE score, which is more likely to be used, gave similar results.

Another contribution of the study is that the authors looked at several different operational definitions of CIND and determined which definitions best predicted later dementia, including AD five years later. The debate still continues with respect to how much risk there is of elderly persons with mild cognitive impairment converting to AD over time. Thus, the perspective provided by this study is of considerable interest, especially as treatment trials are already underway in this group. The base rates in the CSHA study for developing dementia in normal subjects was $15 \%$ over five years. To have any predictive value, the cognitive tests had to show an impairment of at least one standard deviation below age-expected norms, in which case the rates for conversion were $10 \%$ per year to dementia and $6 \%$ per year to $\mathrm{AD}$. These numbers are comparable to other series. Considering the large percentage of individuals with CIND in the elderly population (approximately $30 \%$ of the sample over 65), the absolute numbers are very important. Also the mortality rate for the CIND group over five years was $48.4 \%$, which was significantly greater than the $30.5 \%$ mortality reported for the group with cognitive impairment.

This study is subject to the usual problems of sampling and reflects the limitations inherent in any follow-up study of an elderly cohort five years after initial contact. Those who died or were lost to follow-up constitute a significant subsample and the incidence of dementia cannot obviously be determined accurately in that sample. Pragmatically speaking, however, it is dementia in the survivors that is of concern in terms of the social and economic burden.

In summary, this is a useful population-based study utilizing sound clinical methods. The results provide empirical confirmation for what is intuitively sensible and give solid data to help the clinician triage elderly subjects for further investigation. The study also breaks down mild cognitive impairment into different definitional categories and provides useful information on prognosis. Overall, this is a welcome contribution to the literature.

Sandra Black

Toronto, ON

\section{REFERENCES}

1. Hogan DB, Ebly EM. Predicting who will develop dementia in a cohort of Canadian seniors. Can J Neurol Sci 2000;27:18-24

2. Canadian Study of Health and Aging. Canadian study of health and aging-study methods and prevalence of dementia. Can Med Assoc J 1994; 150:899-913.

3. McDowell I, Kristjansson B, Hill GB, Hebert R. Community screening for dementia - the Mini Mental State Exam (MMSE) and Modified Mini Mental State (3MS) compared. J Clin Epidemiol 1997;(50):377-383.

4. Folstein MF, Folstein SE, McHugh PR. Mini-Mental State - a practical method for grading the cognitive state of patients for the clinician. J Psychiatric Res 1975;12:189-198. 